\title{
Alcohol consumption and labour market participation: a prospective cohort study of transitions between work, unemployment, sickness absence, and social benefits
}

\author{
Maja Bæksgaard Jørgensen ${ }^{1}$ - Jacob Pedersen ${ }^{2}$ Lau Caspar Thygesen ${ }^{1}$ - Cathrine Juel Lau ${ }^{3}$. \\ Anne Illemann Christensen ${ }^{1}$. Ulrik Becker ${ }^{1,4}$. Janne S. Tolstrup ${ }^{1}$
}

Received: 12 October 2017 / Accepted: 15 December 2018 / Published online: 10 January 2019

(c) The Author(s) 2019

\begin{abstract}
The aim of this study was to investigate the association of alcohol consumption and problem drinking on transitions between work, unemployment, sickness absence and social benefits. Participants were 86,417 men and women aged 18-60 years who participated in the Danish National Health Survey in 2010. Information on alcohol consumption (units per week) and problem drinking (CAGE-C score of 4-6) was obtained by questionnaire. The primary outcome was labour market attachment. Information on labour market attachment was obtained from the national administrative registers during a 5-year follow-up period. Using Cox proportional hazards models, we estimated hazard ratios (HR) for transitions between work, unemployment, sickness absence and social benefits. Analyses were adjusted for potential confounders associated with demography, health, and socio-economy. High alcohol consumption and problem drinking was associated with higher probability of unemployment, sickness absence and social benefits among participants employed at baseline compared with participants who consumed 1-6 drinks/week. High alcohol consumption and problem drinking was associated with lower probability of returning to work among participants receiving sickness absence at baseline compared with participants who consumed 1-6 drinks/week and with non-problem drinkers: HRs were 0.75 (0.58-0.98) for 35+ drinks per week and $0.81(0.65-1.00)$ for problem drinking (CAGE-C score of 4-6). Similar trends for weekly alcohol consumption and problem drinking were observed among participants who were unemployed at baseline. In summary, problem drinking has adverse consequences for labour market participation and is associated with higher probability of losing a job and a lower chance of becoming employed again.
\end{abstract}

Keywords Alcohol $\cdot$ Labour market participation $\cdot$ Prospective cohort study

Electronic supplementary material The online version of this article (https://doi.org/10.1007/s10654-018-0476-7) contains supplementary material, which is available to authorized users.

Maja Bæksgaard Jørgensen

mbha@niph.dk

Jacob Pedersen

jpe@ arbejdsmiljoforskning.dk

Lau Caspar Thygesen

lct@niph.dk

Cathrine Juel Lau

Cathrine.Juel.Lau@ @egionh.dk

Anne Illemann Christensen

ach@si-folkesundhed.dk

Ulrik Becker

ulbe@niph.dk
Janne S. Tolstrup

jst@niph.dk

1 National Institute of Public Health, University of Southern Denmark, Studiestræde 6, 1455 Copenhagen, Denmark

2 The National Research Centre for the Working Environment, Lersø Parkallé 105, 2100 Copenhagen, Denmark

3 Center for Clinical Research and Prevention, Bispebjerg and Frederiksberg Hospital, The Capital Region, Copenhagen, Denmark

4 Gastrounit, Medical Division, Copenhagen University Hospital Hvidovre, 2650 Hvidovre, Denmark 


\section{Introduction}

For most people, work is the main source of financial support and ensures that basic needs, such as being able to maintain a home and support a family, are met. Work also has other roles, for example it provides social identity and a feeling of self-worth. Employment status and lifestyle as well as mental and physical health are intimately connected and losing one's job is associated with increased risk of depression [1, 2], cardiovascular disease [3-12], and all-cause mortality [5, 13-16]. Additionally, becoming unemployed is associated with the initiation or extension of unhealthy habits such as smoking and hazardous drinking $[17,18]$. Hence, knowledge of risk factors for job loss and barriers for getting back to work is a great public health concern.

Alcohol is linked to more than 200 diseases and injuries and is the fifth leading risk factor for disease, disability and death worldwide [19, 20]. Harmful alcohol consumption places a vast burden on healthcare and social systems, and in high-and middle-income countries, the cost of this abuse is significant and corresponds to approximately $>1 \%$ of the Gross National Product (GNP) [21]. While associations between alcohol and health outcomes have been extensively studied, less is known about the impact of alcohol on labour market transitions such as becoming unemployed or getting back to work after being unemployed-i.e., downward social selection. Some studies indicate that heavy drinking and alcohol problems are associated with an increased risk of being laid off $[22,23]$ but associations between the full spectrum of alcohol consumption and risk of unemployment are unknown, as is the impact of alcohol on the probability of getting back to work.

In this study, we aim to investigate the association of weekly alcohol consumption and problem drinking on the transition between different labour market endpoints (i.e. work, unemployment, sickness absence and social benefits) in order to identify effective prevention strategies and reduce exclusion within society. We contribute to the literature by using a large, population-based cohort linked to valid administrative registers. Participants were followed for up to 5 years without loss to follow-up while adjustments were made to take account of socio-economic, demographic, and health-related factors were adjusted.

\section{Methods}

This study is based on The Danish National Health Survey 2010 (DNHS-2010) linked to national registers using the unique personal identification number given to all residents in Denmark.

\section{Study design and participants}

The DNHS-2010 is a national health survey conducted in Denmark. DNHS-2010 was based on six mutually exclusive random samples: a national sample and one in each of the five regions of Denmark (the Capital Region of Denmark, Region Zealand, the Region of Southern Denmark, the Central Denmark Region, the North Denmark Region). The sample was randomly selected from the adult population in Denmark, aged 16 years or older, using the Danish Civil Registration System [24]. The sample included 298,850 individuals alive and living in Denmark on 1 January 2010. All selected individuals received a letter that described the purpose of the study and emphasized that participation was voluntary. The letter invited individuals to either fill in the enclosed paper questionnaire or to complete a web questionnaire. Data was collected from February to April 2010. In case of non-response, two postal reminders were sent. The questionnaire was completed by 177,639 individuals, which corresponds to $59.5 \%$ of the total sample. A detailed description of the sampling procedure and design has been published elsewhere [25].

\section{Assessment of alcohol consumption}

Participants were asked how many alcoholic drinks (beer, cider, wine, drinks and spirits) they consumed on each weekday in a typical week. A drink was defined as one beer, one shot or one glass of wine, which corresponded to approximately $12 \mathrm{~g}$ of pure alcohol. The number of drinks per week was calculated and divided into five response categories: no intake, 1-6 drinks/week, 7-13, 14-20, 21-34, and $\geq 35$ drinks/week. The cut-off values were in line with the Danish guidelines for sensible drinking proposed by the Danish Health Authority [26, 27]. Problem drinking was measured by the CAGE-C questionnaire (Cut down, Annoyed, Guilty, Early-morning - Copenhagen) [28], and this study included six items from the questionnaire: (1) Have you, within the past year, felt that you should cut down on your drinking? (2) Have people, within the past year, annoyed you by criticizing your drinking? (3) Have you, within the past year, felt bad or guilty about your drinking? (4) Have you, within the past year, had a drink first thing in the morning to steady your nerves or to get rid of a hangover from time to time? (5) How many days per week do you drink alcohol (positive answer $=4$ days or more)? (6) Do you drink alcohol on weekdays outside mealtimes? The CAGE-C was divided into three categories (no positive answers, 1-3 positive answers, and 4-6 positive answers on the CAGE-C questionnaire) and 4-6 positive answers was defined as problem drinking. 


\section{Assessment of labour market participation}

In Denmark, the labour market is defined by a flexicurity system that includes a high degree of economic compensation and security from the state of Denmark in case of exclusion from the labour market, for example due to unemployment or reduced work ability (security), but is characterized at the same time by a high turnover of jobs and an active (e.g. flexible) labour market policy [29, 30]. Data on labour market participation was obtained from DREAM (The Danish Register-based Evaluation of Marginalization). DREAM contains weekly information for every Danish citizen or resident who receives social transfer payments. The database has been shown to be valid for follow-up of the economic and social consequences of disease [31] and is regularly updated [32]. Labour market outcomes were divided into four categories: work, unemployment, sickness absence, and other social benefit. Selected codes of social transfer payments are available in Table I in the supplementary material. The 'work' state contains all periods during which participants were not receiving any social transfer payments. Social transfer payments obtained from DREAM were divided into three endpoints: unemployment (available to the labour market including on unemployment benefit and social cash-benefits), sickness absence (temporary unavailability to labour market due to health problems), and social benefits (temporary unavailability to labour market). The weekly information was transformed to start and end dates, and we obtained data from Week 5 of 2010 (time of baseline) until Week 5 of 2015. Information on emigration and vital status was obtained from the Danish Civil Registration System [33].

\section{Assessment of potential confounders}

Analyses were adjusted for a priori identified potential confounders known to be associated with both alcohol consumption and labour market outcomes [34, 35]. Information on confounders was obtained in the year of baseline. Three types of confounders were included: demographic, health-related, and socio-economic factors. Demographic confounders: Registers from Statistics Denmark [36] were used to gain information on sex, age, cohabitation status (living alone versus not) and geography (five regions). Healthrelated confounders: The Charlson comorbidity index was calculated from the National Patient Register [37], defined according to 19 selected diseases $[38,39]$ and categorized in two levels (none and any kind of comorbidity). Mental illness and disorders and smoking habits were obtained from the DNHS-2010 questionnaire. Participants reported if they had mental illness and disorders (no, yes-now or previously with current repercussions) and if they were smokers, ex-smokers or current smokers and current smokers reported the number of cigarettes smoked daily. Smoking was categorized in five groups: never smoked, ex-smoker, non-cigarette smoker, 1-14, and 15+ cigarettes/day. Socio-economic confounders: Information on length of education was obtained from Danish education registers [40] and was categorized as less than 10 years, 10-12 years, and more than 12 years of education, which corresponded to primary, upper secondary or vocational and higher education, respectively. Moreover, to adjust for labour market attachment, four factors were included holding information on employment status the year prior to baseline, i.e. amount (\%) of time working, receiving unemployed benefit, sickness absence, and social benefit.

\section{Final study population}

Analyses were restricted to participants aged 18-60 years who were working, in receipt of unemployment or sickness absence, and had intact data sets on alcohol consumption or potential confounders, with the result that 86,417 individuals were eligible for analysis (Fig. 1).

\section{Statistical analysis}

Baseline characteristics of participants were described with median values (5th-95th percentiles) for continuous variables and proportions for categorical variables. All participants were followed from baseline (1 February 2010), when the questionnaires were distributed, to the date of labour market outcomes (mutually exclusive outcomes were included — see Table 2), death, emigration, age limit of 65 years, or the end of follow-up (31 January 2015), whichever came first.

Figure 5 gives a visual illustration of the 12 possible transitions (work, unemployment, sickness absence, and social benefits); however, we did not have sufficient statistical power to study transitions from sickness absence to social benefits and receiving social benefits and so, consequently, a total of eight transitions were analyzed. Each transition was analyzed separately using the Cox Proportional Hazard Model with time since baseline (in days) as the underlying time scale. Reference categories were low alcohol consumption (1-6 drinks per week) or non-problem drinking (4-6 positive answers in the CAGE-C questionnaire). Analyses were adjusted for age, gender, cohabitation status, educational level, Charlson comorbidity index [37], smoking habits, geographic region and labour market participation in the year prior to baseline. The proportional hazards assumption in the Cox model was evaluated by visual inspection of $\log -\log$ plots with no violations detected. Tests for linear and quadratic trends were conducted by treating the median and squared median alcohol consumption within categories as continuous variables. SAS version 9.4 and STATA 
Fig. 1 Chart showing the flow from the Danish National Health Survey (DNHS) 2010 to the final study population

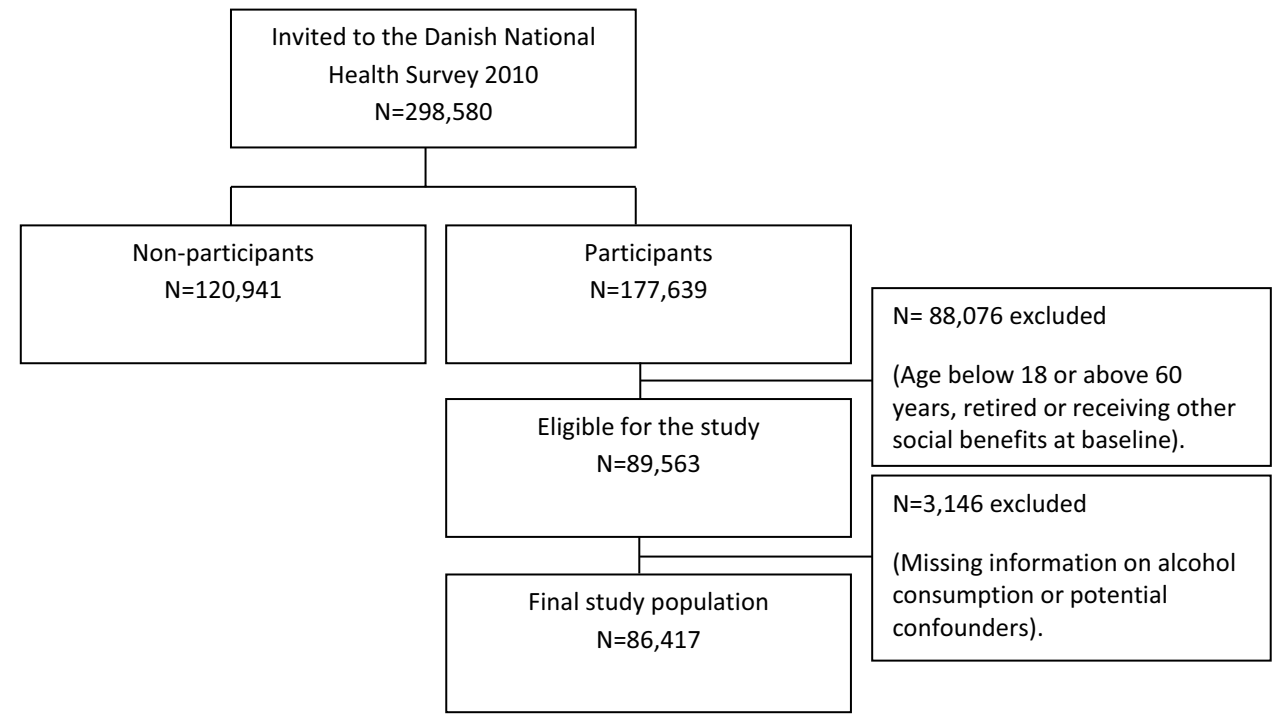

version 15 were used for the statistical analyses. In all tests, $p$ values $<5 \%$ were considered statistically significant.

\section{Sensitivity analyses}

We conducted sensitivity analyses using the Fine-Gray competing risk regression model. However, including competing risks had little impact on results (supplementary materials figures I-III).

\section{Results}

Of the 86,417 participants, 40,912 (47\%) were men (Table 1). The median alcohol consumption was 7.6 (5th-95th, 0-24), 10.0 (5th-95th, 0-36) and 8.3 (5th-95th, 0-30) drinks per week among participants who were working, unemployed and receiving sickness absence. In general, the group of unemployed participants had a shorter education ( $<10$ years) and a more adverse risk factor profile

Table 1 Baseline characteristics of 86,417 participating in the Danish National Health Survey 2010 according to income type

\begin{tabular}{|c|c|c|c|c|}
\hline & \multicolumn{4}{|l|}{ Types of income } \\
\hline & All & $\begin{array}{l}\text { Working }(\mathrm{n}=77,746 \text {, } \\
90.0 \%)\end{array}$ & $\begin{array}{l}\text { Unemployed } \\
(\mathrm{n}=5569,6.4 \%)\end{array}$ & $\begin{array}{l}\text { Sickness } \\
\text { absence }(3102 \text {, } \\
3.6 \%)\end{array}$ \\
\hline $\operatorname{Men}(\mathrm{n}, \%)$ & $40,912(47.3)$ & $36,547(47.0)$ & $3182(57.1)$ & $1183(38.1)$ \\
\hline Age, median yrs (5th-95th) & $43.4(23-58)$ & $43.5(23-58)$ & $42.3(23-59)$ & $45.5(25-59)$ \\
\hline Alcohol, drinks/wk ${ }^{\mathrm{a}}$, median (5th-95th) & $7.7(0-25)$ & $7.6(0-24)$ & $10.0(0-36)$ & $8.3(0-30)$ \\
\hline Problem drinking ${ }^{\mathrm{b}}(\mathrm{n}, \%)$ & $2588(3.0)$ & $2159(2.8)$ & $286(5.1)$ & $143(4.6)$ \\
\hline Primary school education $^{\mathrm{c}}(\mathrm{n}, \%)$ & $16,695(19.3)$ & $13,994(18.0)$ & $1846(33.2)$ & $855(27.6)$ \\
\hline Upper secondary/vocational education $^{\mathrm{d}}(\mathrm{n}, \%)$ & $38,352(44.4)$ & $34,307(44.1)$ & $2606(46.8)$ & $1439(46.4)$ \\
\hline Higher education $^{\mathrm{e}}(\mathrm{n}, \%)$ & $31,370(36.3)$ & $29,445(37.8)$ & $1117(20.1)$ & $808(26.1)$ \\
\hline Current smoker (n, \%) & $21,037(24.3)$ & $17,889(23.0)$ & $2127(38.1)$ & $1021(32.9)$ \\
\hline Living alone (n, \%) & $17,812(20.6)$ & $15,228(19.6)$ & $1808(32.4)$ & $782(25.1)$ \\
\hline Charlson comorbidity index $\geq 1(\mathrm{n}, \%)$ & $4364(5.1)$ & $3543(4.6)$ & $305(5.5)$ & $516(16.6)$ \\
\hline Mental illness and disorders (n, \%) & $1289(1.5)$ & $849(1.10)$ & $150(2.7)$ & $290(9.3)$ \\
\hline
\end{tabular}

Data are presented as median (5th percentile, 95 th percentile) or $\mathrm{n}(\%)$

${ }^{\mathrm{a}}$ One drink corresponds to $12 \mathrm{~g}$ of pure alcohol

${ }^{\mathrm{b}}$ Problem drinking was defined as a CAGE-C score of 4-6

${ }^{\mathrm{c}}<10$ years of education

d $10-12$ years of education

${ }^{\mathrm{e}}>12$ years of education 
(including problem drinking, current smoker and living alone). However, participants receiving sickness absence had the largest proportion of comorbidity and mental illness and disorders compared with participants who were working or unemployed.

\section{Transitions from work to unemployment, sickness absence, and social benefit}

The mean follow-up time varied between the types of labour market participation. At the end of follow-up, a total of 9499 participants had transferred to unemployment, 17,585 to sickness absence and 686 to social benefits among participants working at baseline $(\mathrm{n}=77,746)$ (Table 2).

Among participants who were working at baseline, hazard ratios (HR) of unemployment, sickness absence and social benefit were higher among abstainers and participants with a high alcohol consumption than participants with low consumption (1-6 drinks per week) (Fig. 2). The
HR of unemployment were 1.15 (95\% CI 1.09-1.22) for 0 drinks per week, 1.07 (95\% CI 1.01-1.12) for 7-13 drinks per week, 1.17 (95\% CI 1.09-1.26) for 14-20 drinks per week, 1.33 (95\% CI 1.19-1.48) for 21-27 drinks per week, 1.35 (95\% CI 1.17-1.55) for 28-34 drinks per week, and 1.44 (95\% CI 1.28-1.63) for 35+ drinks per week. For the transition between work and sickness absence, the HR was lower among those who consumed 7-13 drinks per week with an HR of 0.93 (95\% CI 0.89-0.97).

\section{Transitions from unemployment to work, sickness absence and other social benefits}

Among participants unemployed at baseline $(\mathrm{n}=5569)$, a total of 4517 participants had returned to work, 767 had transferred to sickness absence and 182 had transferred to social benefits at the end of follow-up (Table 2). Heavy alcohol consumption of $35+$ drinks per week was associated with a higher risk of transfer to sickness absence among

Table 2 Number (\%) and mean follow-up time (5th-95th percentiles, in years) for transitions between work, unemployment and sickness absence at baseline to work, unemployment, sickness absence and

social benefits at follow up, in 86,417 participants from the Danish National Health Survey 2010

\begin{tabular}{llllll}
\hline & \multicolumn{2}{l}{ Types of labour market participation } \\
\cline { 2 - 6 } & To work & To unemployment & To sickness absence & To social benefits & To censoring \\
\hline From work & - & $9499(12.2)$ & $17,585(22.6)$ & $686(0.9)$ & $49,976(64.3)$ \\
$77,746(90.0)$ & & 1.8 years $(0.06-4.56)$ & 1.9 years $(0.08-4.62)$ & 1.5 years $(0.04-4.16)$ & $4.8(3.41-5.0)$ \\
From unemployment & $4517(81.1)$ & - & $767(13.7)$ & $182(3.3)$ & $103(1.9)$ \\
$5569(6.4)$ & 0.4 years $(0.04-1.34)$ & & 0.5 years $(0.02-1.76)$ & 0.5 years $(0.02-1.49)$ & 0.6 years $(0.04-2.17)$ \\
From sickness absence & $2302(74.2)$ & $442(14.3)$ & - & $71(2.3)$ & $287(9.2)$ \\
$3102(3.6)$ & 0.4 years $(0.02-1.42)$ & 0.4 years $(0.02-1.42)$ & & 1.1 years $(0.08-2.72)$ & 0.9 years $(0.15-1.99)$ \\
\hline
\end{tabular}

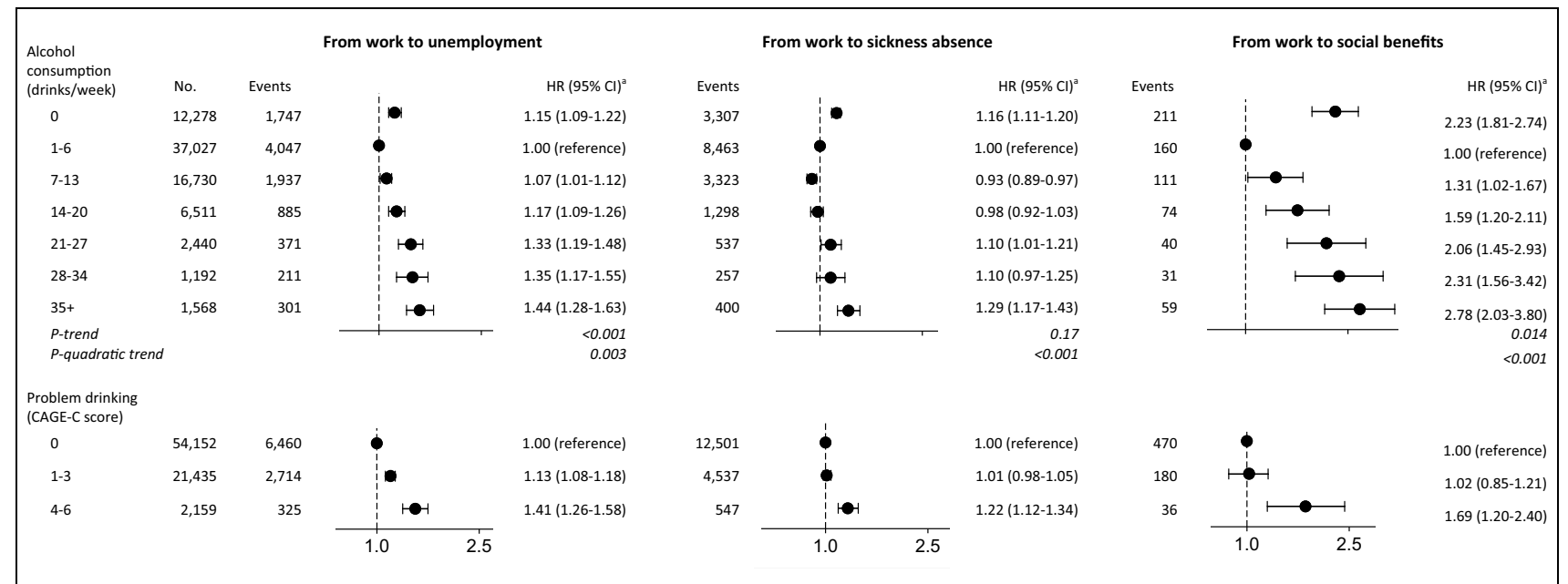

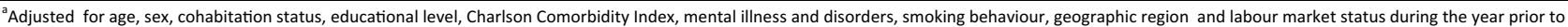
baseline

Fig. 2 Hazard ratios and 95\% confidence intervals for transitions from work to unemployment, sickness absence or social benefits according to weekly alcohol consumption and problem drinking in 77,746 participating in the Danish National Health Survey 2010 
participants who were unemployed at baseline. When compared with participants who drank 1-6 drinks per week, the HR among drinkers of $35+$ drinks per week was 1.58 (95\% CI 1.14-2.18). Additionally, the HR of transfer from unemployment to social benefit was also higher among participants with high alcohol consumption of $28+$ drinks per week when compared with participants who consumed 1-6 drinks per week (Fig. 3).

\section{Transitions from sickness absence to work and unemployment}

A total of 2302 participants had returned to work and 442 transferred to unemployment (Table 2). Among participants receiving sickness absence at baseline, we found a lower HR of returning to work among abstainers and consumers of $35+$ drinks per week with an HR of 0.78 (95\% CI 0.70-0.87) and 0.75 (95\% CI 0.58-0.98) when compared with those who had 1-6 drinks per week. For the transition between sickness absence and unemployment, the HR was higher among consumers of 28-34 drinks per week with an HR of 1.74 (95\% CI 1.01-3.00) (Fig. 4).

\section{Problem drinking and transitions between work, employment, sickness absence and social benefits}

Figure 5 shows the HR for transitions between all four endpoints according to problem drinking (CAGE-C score of 4-6). Problem drinking was associated with a higher HR of unemployment, sickness absence and social benefits among participants who were working at baseline with an HR of 1.41 (95\% CI 1.26-1.58), 1.22 (95\% CI 1.12-1.34), and
1.69 (95\% CI 1.20-2.40) when compared with non-problem drinkers.

A significantly lower probability of returning to work was observed among participants who were problem drinkers and unemployed at baseline compared with non-problem drinkers with an HR of 0.86 (95\% CI 0.74-0.99). Furthermore, problem drinking was associated with a higher HR of transfer to sickness absence and social benefits among participants who were unemployed at baseline compared with non-problem drinkers.

\section{Discussion}

Results from this study showed that high amounts of weekly alcohol consumption and problem drinking were associated with an increased HR of unemployment, sickness absence and social benefits among participants employed at baseline. Moreover, results showed that high amounts of weekly alcohol consumption and problem drinking were associated with lower probability of returning to work after being unemployed or receiving sickness absence and increased the HR of transfer to social benefits (sickness absence and social benefits). Analyses of all eight transitions showed that abstainers had a similar HR to individuals with heavy alcohol consumption.

\section{Strengths and limitations}

The main strengths of this prospective cohort study are the large study sample and virtually complete follow-up for labour market outcomes through linkage to nationwide

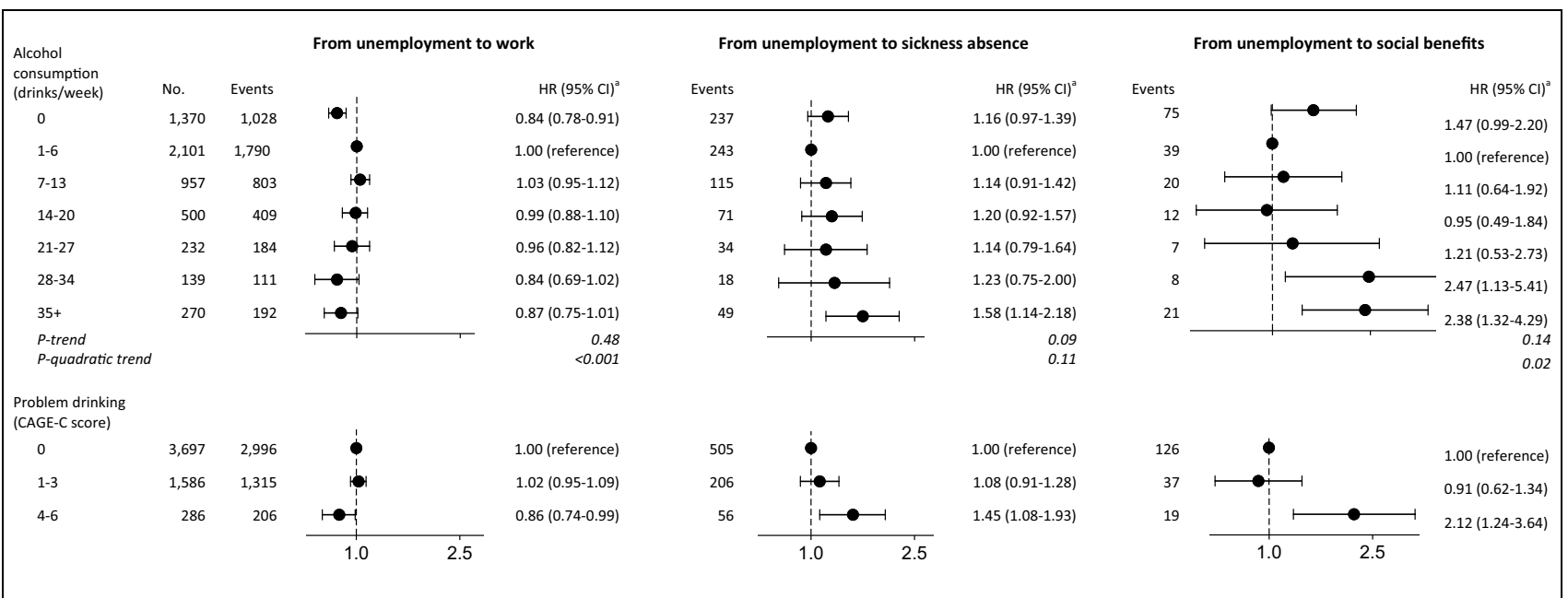

aAjusted for age, sex, cohabitation status, educational level, Charlson Comorbidity Index, mental illness and disorders, smoking behaviour, geographic region and labour market status during the year prior to baseline

Fig. 3 Hazard ratios and 95\% confidence intervals for transitions from work unemployment to work, sickness absence and social benefit by weekly alcohol consumption and CAGE-C in 5569 participating in the Danish National Health Survey 2010 


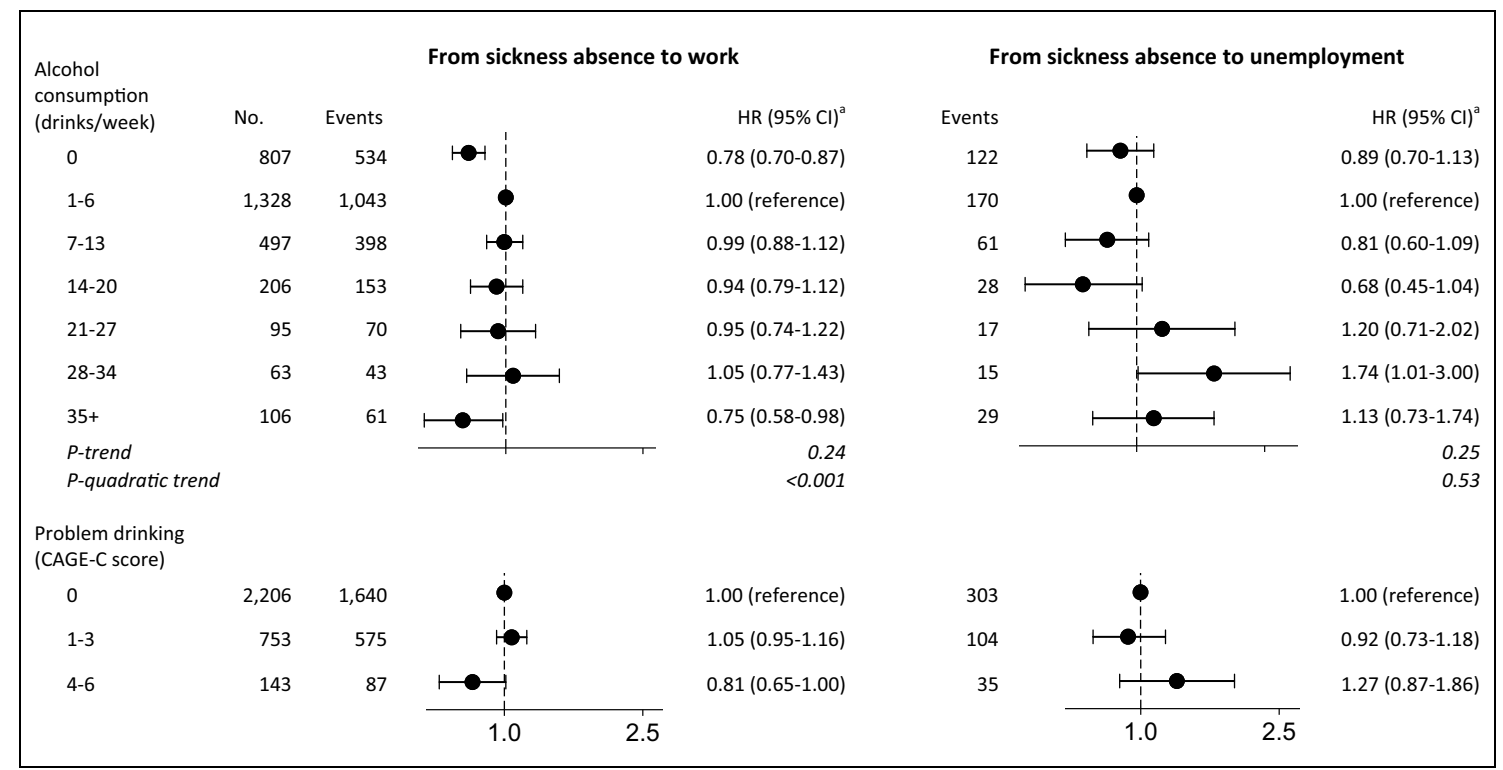

${ }^{a}$ Adjusted for age, sex, cohabitation status, educational level, Charlson Comorbidity Index, mental illness and disorders, smoking behaviour, geographic region and labour market status during the year prior to baseline

Fig. 4 Hazard ratios and 95\% confidence intervals for transitions from sickness absence to work, unemployment and social benefit by weekly alcohol consumption in 3102 participating in the Danish National Health Survey 2010

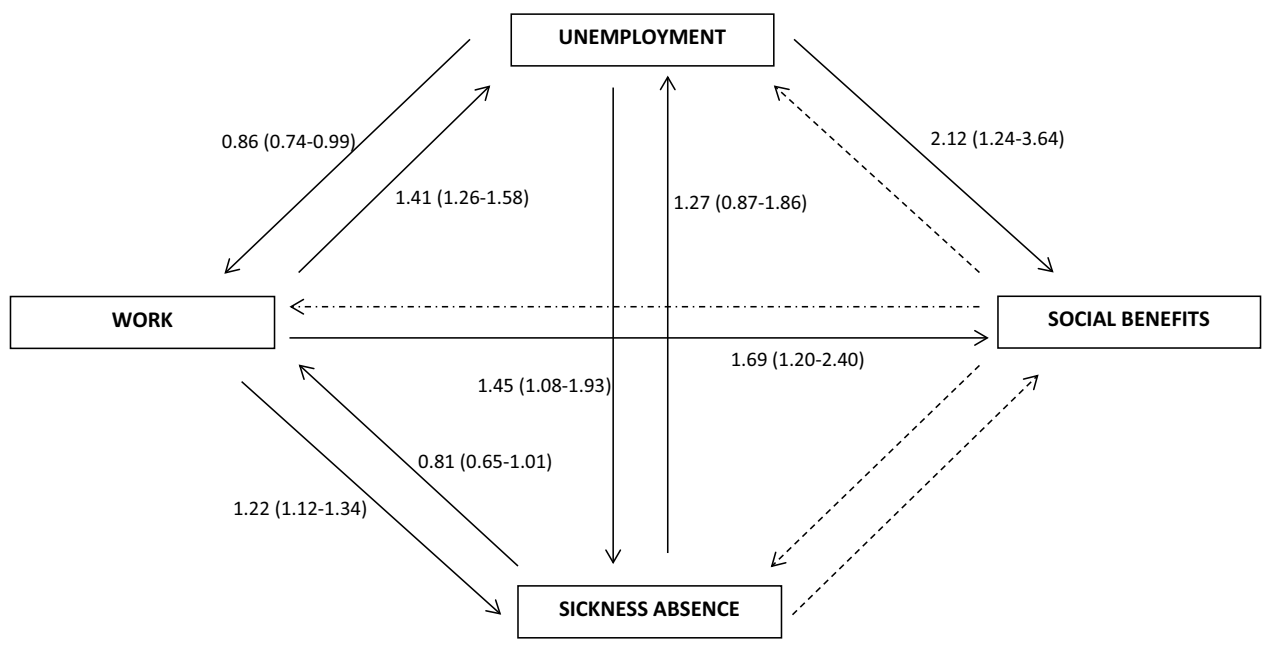

Fig. 5 Hazard ratios and 95\% confidence intervals for transitions between work, unemployment and sickness absence at baseline to work, unemployment, sickness absence and social benefits at followup according to problem drinking (CAGE-C score of 4-6). Reference group was no problem drinking. Dashed arrows represent transitions

registers. A further strength is the ability to examine labour market participants across a wide range of alcohol consumption and problem drinking.

Several limitations, however, are evident in this study. Firstly, measures of alcohol consumption were self-reported and likely to be underestimated [41]. Even so, the methods applied in this study are generally regarded to be valid and that was not possible to analyze, due to low power. The eight transitions were adjusted for age, gender, cohabitation status, educational level, Charlson Comorbidity Index, mental illness and disorders, smoking behaviour, geographic region and labour market status during the year prior to baseline

found suitable for use in epidemiological studies [42] as results are also similar using different methods of estimating alcohol use. Secondly, we were unable to separate abstainers and former drinkers due to limitations in the DNHS-2010 questionnaire. This information would have been valuable as individuals may have altered their weekly alcohol consumption in response to health conditions and this might explain 
the similar risk estimates among abstainers and heavy drinkers [43-45]. This phenomenon is often referred to as the 'sick quitter' effect or former drinker's bias [46, 47] and as a consequence, all analyses were adjusted for comorbidities (the Charlson comorbidity index) and mental illness and disorders to account for comorbidity. However, this issue was not a problem when analyzing problem drinking. Here the lowest risk estimates belong to individuals categorized as non-problem drinkers (no positive answers on the CAGE-C questionnare).

Thirdly, we used the DREAM register to obtain information on labour market participants. It is important to note that invisible unemployment could occur (e.g. if an unemployed individual is not in receipt of any social transfer benefit) [48]. Additionally, no record was made to distinguish between individuals who are receiving sickness benefit but are still employed and individuals who are receiving sickness benefit but are unemployed. This DREAM register has previously been proven suitable for analyses of labour market consequences [32]. Fourthly, although we made adjustments for multiple potential confounders, we cannot rule out the possibility of residual confounding or unmeasured confounding. Factors such as occupational status, income, or other risk determinants might also be associated with exposure and endpoints. A proxy variable for occupational status and income was available through information on education found in the Danish education registers.

Fifthly, the study population was restricted to participants aged 18-60 years, which is a relatively large age range. We conducted sensitivity analyses that excluded participants aged 55-60 years, as the older population could have an increased HR of sickness absence or differ in other ways from the general population. This exclusion did not substantially change the estimates. Sixthly, only $59.5 \%$ of those invited participated and the people who declined may have had different risk profiles to those who chose to participate. A recently conducted Danish study compared cause-specific mortality and morbidity among survey respondents and nonrespondents from the Danish Health and Morbidity Survey population collected in 2000 and 2005 [49]. This study found that non-respondents in Denmark have an increased hazard ratio of alcohol, drug and smoking-related mortality and morbidity when compared with respondents [49]. This may have affected the results and some caution should be taken when interpreting the findings.

A final limitation is that the interpretation of results may be confounded by unknown or unmeasured factors associated with exposure and endpoint (labour market participation), which could have biased the risk estimates [35]. It is likely that both exposure and endpoint are associated with other physical, social, and behavioural risk factors not included in the analyses, such as impulsivity, lower cognitive ability, or, socioeconomic disadvantages occurring early in life or genes [16, 49-51].We included information on previously applied confounders; [22] but we were unable to apply a life-course perspective because information was not available in this study and confounding due to unknown or unmeasured factors and residual confounding due to broad categorization cannot be excluded. Previous studies suggest that this is only a problem if the confounding factors are strongly associated with endpoints and distributed differently between the exposure groups [52].

\section{Comparison with other studies}

The mechanisms by which alcohol could impact on labour market participants are complex and not entirely understood $[22,53]$. In the literature it is suggested that moderate alcohol consumption has a beneficial effect on labour outcomes [43, 44, 54-57]; however, these findings are often disputed due to the potential of e.g. selection or former drinker bias [53]. Previous literature has also suggested that heavy alcohol consumption may have an adverse effect on labour market participants in terms of loss of productivity and ability to work. [23, 58-63] In the short term, heavy alcohol consumption may have social and legal consequences, lead to hangovers from binge drinking, and increase absenteeism from work. Additionally, heavy alcohol consumption may cause health problems in the long term [23, 64], creating a vicious circle which may affect labour market participation [16].

To the best of our knowledge, no previous studies have investigated the transitions between different labour market outcomes by weekly alcohol consumption and problem drinking as we have done in this study. The strong evidence base for alcohol consequences on disease contrasts with relatively few studies on alcohol consumption and labour market outcomes. Previous studies have been restricted to single labour market outcomes such as employment, working hours, wages and earnings, absenteeism and unemployment [22]. Consistent with our findings, the literature generally suggests that a non-linear association is present (e.g. moderate alcohol consumers have higher wages and earnings compared to abstainers and heavy alcohol users) [22, 23, 43, $44,54,55,57]$.

Other studies have examined whether alcohol consumption is related to labour market participation and attachment [23]. Some studies indicate that heavy alcohol consumption increases the probability of being unemployed. [23, 58-63] This is in contrast to other studies that could not find a statistically significant relationship between alcohol consumption and labour market participation $[64,65]$. The difference between the results may be partly explained by differences in the sampling methods, study population, data quality and design as well as country-specific characteristics. For example, the majority of studies have been conducted in countries 
that are not comparable to the Danish labour market conditions and welfare system.

\section{Implications and future research}

Harmful alcohol consumption remains a key public health concern. Alcohol-attributable factors play a major part in producing health inequalities. Having a job and good health are intimately connected. Job loss has an adverse effect on health behaviour as well as on mental and physical healthwhich could create a vicious circle of growing health inequalities. The available evidence sheds light on a hidden consequence of alcohol consumption. By drawing attention to the field, we hope to stimulate more research that will inform policymakers about the magnitude of the problems.

Additional research should elucidate whether observed associations in this study could be reproduced in other countries. In order to prevent adverse consequences from alcohol consumption, more research is needed to investigate the mechanisms behind the underlying causes. More knowledge might help to clarify public health aims, lead to an increased focus and provide guidance on new ways to target individuals within and outside the labour market. It would also be interesting to study the inverse relationship between unemployment and heavy drinking or alcohol problems.

\section{Conclusion}

In this large prospective cohort study of the general Danish population, the results suggest that high alcohol consumption and problem drinking increases the risk of leaving the labour market and is a barrier to re-employment.

Acknowledgements The authors like to thank the TrygFonden, Denmark for financing this work. The Danish Health Survey 2010 was funded by the Capital Region of Denmark, Region Zealand, the Region of Southern Denmark, the Central Denmark Region, the North Denmark Region, the Danish Ministry of Interior and Health and the Danish National Institute of Public Health at the University of Southern Denmark. We thank the participants of The Danish National Health Survey 2010 for their time answering the questionnaires and their cooperation. The funding sources had no role in the study design, analyses, decision to publish or preparation of the manuscript.

Funding This study was funded by TrygFonden, Denmark.

\section{Compliance with ethical standards}

Conflict of interest The authors declare that they have no conflict of interest.

Ethical standards The study did not require any ethical approval. All participants in The Danish Health Survey 2010 gave infomed consent before taking part in the study.
Open Access This article is distributed under the terms of the Creative Commons Attribution 4.0 International License (http://creativeco mmons.org/licenses/by/4.0/), which permits unrestricted use, distribution, and reproduction in any medium, provided you give appropriate credit to the original author(s) and the source, provide a link to the Creative Commons license, and indicate if changes were made.

\section{References}

1. Mossakowski KN. The influence of past unemployment duration on symptoms of depression among young women and men in the United States. Am J Public Health. 2009;99(10):1826-32.

2. Berchick ER, Gallo WT, Maralani V, Kasl SV. Inequality and the Association between involuntary job loss and depressive symptoms. Soc Sci Med. 2012;75(10):1891-4. https://doi. org/10.1016/j.socscimed.2012.07.024.

3. Gallo WT, Bradley EH, Falba TA, et al. Involuntary job loss as a risk factor for subsequent myocardial infarction and stroke: findings from the Health and Retirement Survey. Am J Ind Med. 2004;45(5):408-16.

4. Gallo WT, Teng HM, Falba TA, Kasl SV, Krumholz HM, Bradley EH. The impact of late career job loss on myocardial infarction and stroke: a 10 year follow up using the health and retirement survey. Occup Environ Med. 2006;63(10):683-7.

5. Garcy AM, Vagero D. The length of unemployment predicts mortality, differently in men and women, and by cause of death: a six year mortality follow-up of the Swedish 1992-1996 recession. Soc Sci Med (1982). 2012;74(12):1911-20.

6. Olesen K, Rugulies R, Rod NH, Bonde JP. Does retirement reduce the risk of myocardial infarction? A prospective registry linkage study of 617511 Danish workers. Int J Epidemiol. 2014;43(1):160-7.

7. Dupre ME, George LK, Liu G, Peterson ED. The cumulative effect of unemployment on risks for acute myocardial infarction. Arch Intern Med. 2012;172(22):1731-7.

8. Noelke C, Avendano M. Who suffers during recessions? Economic downturns, job loss, and cardiovascular disease in older Americans. Am J Epidemiol. 2015;182(10):873-82.

9. Kang MY, Kim HR. Association between voluntary/involuntary job loss and the development of stroke or cardiovascular disease: a prospective study of middle-aged to older workers in a rapidly developing Asian country. PLoS ONE. 2014;9(11):e113495.

10. Franks PJ, Adamson C, Bulpitt PF, Bulpitt CJ. Stroke death and unemployment in London. J Epidemiol Community Health. 1991;45(1):16-8.

11. Moon JR, Glymour MM, Subramanian SV, Avendano M, Kawachi I. Transition to retirement and risk of cardiovascular disease: prospective analysis of the US health and retirement study. Soc Sci Med (1982). 2012;75(3):526-30.

12. Eshak ES, Honjo $\mathrm{K}$, Iso $\mathrm{H}$, et al. Changes in the employment status and risk of stroke and stroke types. Stroke. 2017;48(5):1176-82.

13. Martikainen PT, Valkonen T. Excess mortality of unemployed men and women during a period of rapidly increasing unemployment. Lancet (London, England). 1996;348(9032):909-12.

14. Dahlgren D, Whitehead M. European strategies for tackling social inequalities in health: levelling up Part 2 World Health Organization (WHO). http://www.thehealthwell.info/node/91930 (2007). Accessed 4 Jan 2019.

15. Diderichsen F, Evens T, Whitehead M. The Social Basis of Disparities in Health. In: Evans T, Whitehead M, Diderichsen F, Bhuiya A, Wirth M, editors. Challenging inequities in health From ethics to action. Oxford: Oxford University Press; 2001. p. 13-23. 
16. Diderichsen F. Health inequalities - a challenge for the social investment welfare state. Nordic Welfare Res. 2016;1:43-54.

17. Bosque-Prous M, Espelt A, Sordo L, Guitart AM, Brugal MT, Bravo MJ. Job loss, unemployment and the incidence of hazardous drinking during the late 2000 s recession in europe among adults aged 50-64 years. PLoS ONE. 2015;10(10):e0140017.

18. Waters LE. Coping with unemployment: a literature review and presentation of a new model. IJMR. 2000;2:169-82. https://doi. org/10.1111/1468-2370.00036.

19. World Health Organization. Global status report on alcohol and health 2014. Geneva: World Health Organization; 2014.

20. Lim SS, Vos T, Flaxman AD, et al. A comparative risk assessment of burden of disease and injury attributable to 67 risk factors and risk factor clusters in 21 regions, 1990-2010: a systematic analysis for the Global Burden of Disease Study 2010. Lancet (London, England). 2012;380(9859):2224-60.

21. Rehm J, Mathers C, Popova S, Thavorncharoensap M, Teerawattananon Y, Patra J. Global burden of disease and injury and economic cost attributable to alcohol use and alcohol-use disorders. Lancet (London, England). 2009;373(9682):2223-33.

22. Henkel D. Unemployment and substance use: a review of the literature (1990-2010). Curr Drug Abuse Rev. 2011;4(1):24.

23. Bockerman P, Hyytinen A, Maczulskij T. Alcohol consumption and long-term labor market outcomes. Health Econ. 2015;26(3):275-91.

24. Pedersen CB. The Danish Civil Registration System. Scand J Public Health. 2011;39(7 Suppl):22-5.

25. Christensen AI, Ekholm O, Glumer C, et al. The Danish National Health Survey 2010. Study design and respondent characteristics. Scand J Public Health. 2012;40(4):391-7.

26. Danish Health Authority. Health and lifestyle: Alcohol https:// www.sst.dk/en/health-and-lifestyle/alcohol2009. 20 Feb 2017.

27. Kalinowski A, Humphreys K. Governmental standard drink definitions and low-risk alcohol consumption guidelines in 37 countries. Addiction (Abingdon, England). 2016;111(7):1293-8.

28. Zierau F, Hardt F, Henriksen JH, et al. Validation of a self-administered modified CAGE test (CAGE-C) in a somatic hospital ward: comparison with biochemical markers. Scand J Clin Lab Invest. 2005;65(7):615-22.

29. Dølvig J, Fløtten T, Hippe J, Jordfald B. The Nordic model toward 2030. A new chapter? (2014). https://www.fafo.no/image s/pub/2015/20412.pdf. Accessed 4 Jan 2019.

30. Carlsen K, Harling H, Pedersen J, et al.The transition between work, sickness absence and pension in a cohort of Danish colorectal cancer survivors.BMJ Open 2013;3:e02259. https://doi. org/10.1136/bmjopen-2012-002259.

31. Hjollund NH, Larsen FB, Andersen JH. Register-based followup of social benefits and other transfer payments: accuracy and degree of completeness in a Danish interdepartmental administrative database compared with a population-based survey. Scand J Public Health. 2007;35(5):497-502.

32. Arbejdsmarkedsstyrelsen. DREAM version 36. Manual. Copenhagen: Arbejdsmarkedstyrelsen; 2016.

33. Schmidt M, Pedersen L, Sorensen HT. The Danish Civil Registration System as a tool in epidemiology. Eur J Epidemiol. 2014;29(8):541-9.

34. Greenland S, Morgenstern H. Confounding in health research. Annu Rev Public Health. 2001;22:189-212.

35. Rothman KJ, Greenland S. Modern epidemiology. Washington: Lippincott-Raven; 1998.

36. Statistics Denmark. The Danish system for access to micro data (2017). https://www.google.dk/ $\mathrm{url}$ ? $\mathrm{sa}=\mathrm{t} \& \mathrm{rct}=\mathrm{j} \& \mathrm{q}=\& \mathrm{esrc}=\mathrm{s} \&$ source $=$ web $\& \mathrm{~cd}=1 \& \mathrm{ved}=2 \mathrm{ahUK}$ Ewie0ebRqdTfAhWO2KQKHUESCz8QFjAAegQICBAC $\&$ url $=\mathrm{https} \% 3 \mathrm{~A} \% 2 \mathrm{~F} \% 2 \mathrm{Fwww} . \mathrm{dst} . \mathrm{dk} \% 2 \mathrm{Fext} \% 2 \mathrm{~F} 545$ $2354440 \% 2 \mathrm{~F} 0 \% 2 \mathrm{Fisrae} 12016 \% 2 \mathrm{FAnn}$ ex-B5-15-Intro
duction-to-the-Danish-system-for-acces s-to-microdata-pdf\&usg=AOvVaw1mGbxMdV587ExmDub7MvFg(2014).

37. Lynge E, Sandegaard JL, Rebolj M. The Danish national patient register. Scand J Public Health. 2011;39(7 Suppl):30-3.

38. Charlson ME, Pompei P, Ales KL, MacKenzie CR. A new method of classifying prognostic comorbidity in longitudinal studies: development and validation. J Chronic Dis. 1987;40(5):373-83.

39. Thygesen SK, Christiansen CF, Christensen S, Lash TL, Sorensen HT. The predictive value of ICD-10 diagnostic coding used to assess Charlson comorbidity index conditions in the populationbased Danish National Registry of Patients. BMC Med Res Methodol. 2011;11:83.

40. Jensen VM, Rasmussen AW. Danish education registers. Scand J Public Health. 2011;39(7 Suppl):91-4.

41. de Leeuw E, Hox J. International handbook of survey methodology. New York: Taylor \& Francis Group; 2008.

42. Ekholm O, Strandberg-Larsen K, Christensen K, Gronbaek M. Comparison of assessment methods for self-reported alcohol consumption in health interview surveys. Eur J Clin Nutr. 2008;62(2):286-91.

43. French MT, Zarkin GA. Is moderate alcohol use related to wages? Evidence from four worksites. J Health Econ. 1995;14(3):319-44.

44. Heien D. The relationship between alcohol consumption and earnings. J Stud Alcohol. 1996;57(5):536-42.

45. Kaila-Kangas L, Kivekas T, Laitinen J, et al. Abstinence and current or former alcohol use as predictors of disability retirement in Finland. Scand J Public Health. 2015;43(4):373-80.

46. Fillmore KM, Stockwell T, Chikritzhs T, Bostrom A, Kerr W. Moderate alcohol use and reduced mortality risk: systematic error in prospective studies and new hypotheses. Ann Epidemiol. 2007;17(5 Suppl):S16-23.

47. Shaper AG, Wannamethee G, Walker M. Alcohol and mortality in British men: explaining the U-shaped curve. Lancet (London, England). 1988;2(8623):1267-73.

48. Pedersen J, Bjorner JB, Burr H, Christensen KB. Transitions between sickness absence, work, unemployment, and disability in Denmark 2004-2008. Scand J Work Environ Health. 2012;38(6):516-26

49. Christensen AI, Ekholm O, Gray L, Glumer C, Juel K. What is wrong with non-respondents? Alcohol-, drug- and smokingrelated mortality and morbidity in a 12-year follow-up study of respondents and non-respondents in the Danish Health and Morbidity Survey. Addiction (Abingdon, England). 2015;110(9):1505-12.

50. Blas E, Kurup A. In: Blas E, Kurup AS, editors. Equity, social determinants and public health programmes. Geneva: World Health Association; 2010. http://www.who.int/iris/handle/10665 144289. Accessed 4 Jan 2018.

51. Khemiri L, Kuja-Halkola R, Larsson H, Jayaram-Lindström N. Genetic overlap between impulsivity and alcohol dependence: a large-scale national twin study. Psychol Med. 2016;46(5):1091-102.

52. Latvala A, Kuja-Halkola R, D'Onofrio B, Larsson H, Lichtenstein P. Cognitive ability and risk for substance misuse in men: genetic and environmental correlations in a longitudinal nation-wide family study. Addiction (Abingdon, England). 2016;111:1814-22.

53. Devaux M. Social disparities in alcohol drinking. In: Sassi F, editor. Tackling harmful alcohol use-economics and public health policy. Paris: OECD; 2015.

54. Barrett G. The effect of alcohol consumption on earnings. Econ Rec. 2002;78:79-96.

55. Hamilton V, Hamilton B. Alcohol and earnings: does drinking yield a wage premium? Can J Econ. 1997;30:135-51.

56. Lee YL. Wage effects of drinking in Australia. Aust Econ Rev. 2003;36:265-82. 
57. Zarkin GA, French MT, Mroz T, Bray JW. Alcohol use and wages: new results from the National Household Survey on Drug Abuse. J Health Econ. 1998;17(1):53-68.

58. MacDonald Z, Shields MA. The impact of alcohol consumption on occupational attainment in England. Economica. 2001;68:427-53.

59. MacDonald Z, Shields MA. Does problem drinking affect employment? Evidence from England. Health Econ. 2004;13(2):139-55.

60. Johansson E, Alho H, Kiiskinen U, Poikolainen K. The association of alcohol dependency with employment probability: evidence from the population survey 'Health 2000 in Finland'. Health Econ. 2007;16(7):739-54.

61. Paljarvi T, Martikainen P, Pensola T, Leinonen T, Herttua K, Makela P. Life course trajectories of labour market participation among young adults who experienced severe alcohol-related health outcomes: a retrospective cohort study. PLoS ONE. 2015;10(5):e0126215.

62. Booth BM, Feng W. The impact of drinking and drinking consequences on short-term employment outcomes in at-risk drinkers in six southern states. J Behav Health Serv Res. 2002;29(2):157-66.
63. Dooley D, Catalano R, Hough R. Unemployment and alcohol disorder in 1910 and 1990: drift versus social causation. J Occup Organ Psychol. 1992;65:277-90.

64. Mullahy J, Sindelar J. Employment, unemployment, and problem drinking. J Health Econ. 1996;15(4):409-34.

65. Feng W, Zhou W, Butler JS, Booth BM, French MT. The impact of problem drinking on employment. Health Econ. 2001;10(6):509-21.

Publisher's Note Springer Nature remains neutral with regard to jurisdictional claims in published maps and institutional affiliations. 